\title{
Reciprocal Anatomical Relationship between Primary Sensory and Prefrontal Cortices in the Human Brain
}

\author{
Chen Song, ${ }^{1,2}$ Dietrich Samuel Schwarzkopf, ${ }^{1,2}$ Ryota Kanai, ${ }^{1}$ and Geraint Rees ${ }^{1,2}$ \\ ${ }^{1}$ Institute of Cognitive Neuroscience, University College London, London WC1N 3AR, United Kingdom, and ${ }^{2}$ Wellcome Trust Centre for Neuroimaging, \\ University College London, London WC1N 3BG, United Kingdom
}

\begin{abstract}
The human brain exhibits remarkable interindividual variability in cortical architecture. Despite extensive evidence for the behavioral consequences of such anatomical variability in individual cortical regions, it is unclear whether and how different cortical regions covary in morphology. Using a novel approach that combined noninvasive cortical functional mapping with whole-brain voxel-based morphometric analyses, we investigated the anatomical relationship between the functionally mapped visual cortices and other cortical structures in healthy humans. We found a striking anticorrelation between the gray matter volume of primary visual cortex and that of anterior prefrontal cortex, independent from individual differences in overall brain volume. Notably, this negative correlation formed along anatomically separate pathways, as the dorsal and ventral parts of primary visual cortex showed focal anticorrelation with the dorsolateral and ventromedial parts of anterior prefrontal cortex, respectively. Moreover, a similar inverse correlation was found between primary auditory cortex and anterior prefrontal cortex, but no anatomical relationship was observed between other visual cortices and anterior prefrontal cortex. Together, these findings indicate that an anatomical trade-off exists between primary sensory cortices and anterior prefrontal cortex as a possible general principle of human cortical organization. This new discovery challenges the traditional view that the sizes of different brain areas simply scale with overall brain size and suggests the existence of shared genetic or developmental factors that contributes to the formation of anatomically and functionally distant cortical regions.
\end{abstract}

\section{Introduction}

Human neocortex is divided into anatomically and functionally distinct cortical regions (Pallas, 2001), such as primary sensory cortices for basic sensory processing and prefrontal cortex for complex decision making. The relative expansion and contraction of these cortical regions along the path of primate evolution reflects their importance in generating behavioral complexity (Schoenemann, 2006). For example, the marked expansion of anterior prefrontal cortex indicates its key role in high-order cognitive functions unique to humans (Semendeferi et al., 2001). Likewise, the variability in size of individual cortical regions within a single species such as humans is representative of their functionality in associated cognitive domains: people with bigger anterior prefrontal cortex exhibit better introspective ability (Fleming et al., 2010), and those with smaller primary visual cortex experience stronger visual illusions (Schwarzkopf et al., 2011). But in contrast to this growing interest in behavioral consequences of anatomical variability in single cortical region, there has until now been

\footnotetext{
Received Jan. 18, 2011; revised April 15, 2011; accepted May 11, 2011.

Author contributions: C.S., D.S.S., R.K., and G.R. designed research; C.S., D.S.S., and R.K. performed research; C.S. analyzed data; C.S., D.S.S., R.K., and G.R. wrote the paper.

This work was supported by the Brain Research Trust (C.S.), Japan Society for the Promotion of Science (R.K.), and the Wellcome Trust (G.R., D.S.S.). We thank Haishan Yao and Cheng Chen for comments on this manuscript.

The authors declare no competing financial interests.

Correspondence should be addressed to Chen Song, Institute of Cognitive Neuroscience, University College London, 17 Queen Square, London WC1N 3AR, UK. E-mail: chen.song.09@ucl.ac.uk.

DOI:10.1523/JNEUROSCI.0308-11.2011

Copyright (C) 2011 the authors $\quad 0270-6474 / 11 / 319472-09 \$ 15.00 / 0$
}

little investigation on the anatomical consequences of such interindividual variability for other cortical regions.

One key problem in investigating the anatomical relationship between different human cortical areas is that boundaries between most cortical areas are difficult to delineate unambiguously and noninvasively. Thus, previous studies have been limited to using regions of interest (ROIs) based on brain atlases (Mechelli et al., 2005; Hagmann et al., 2008). These regions of interest defined on probabilistic grounds do not necessarily correspond well with individual locations of cytoarchitectonic area boundaries and may thus dramatically underrepresent the anatomical variability in the whole cortical area across different individuals. In contrast, the locations and volumes of early visual cortices can be measured accurately and reproducibly using retinotopic mapping (Sereno et al., 1995). The size of primary visual cortex (V1) varies by as much as threefold among healthy adults (Stensaas et al., 1974) and is correlated with the size of other subcortical visual structures (Andrews et al., 1997; Dougherty et al., 2003). However, how changes in V1 size might be associated with variability in size of the rest of cortex remains unclear. One possibility is that individuals with bigger V1 also show larger cortical regions in general. Alternatively, the sizes of functionally segregated cortical regions may be uniquely determined with no shared variance. To investigate the principles of anatomical covariance in human brain, we therefore used functionally mapped visual cortices as seed regions and systematically explored the consequences of their anatomical variability for all other cortical regions. 
To anticipate our findings, the sizes of different cortical areas did not necessarily scale with each other. While we found a positive correlation between gray matter volumes of primary visual cortex and primary auditory cortex, we found a strong anatomical trade-off between the volumes of primary visual or auditory cortex and anterior prefrontal cortex. This discovery indicates that the development of functionally and anatomically distinct cortical regions are nonetheless mediated by common factors and hints toward an opposing interplay between basic sensory and high-order cognitive functions in humans.

\section{Materials and Methods}

The gray matter volumes of early visual areas (V1, V2, V3) were measured using standard retinotopic mapping (Sereno et al., 1995) in a group of 30 healthy human participants. Structural MRI images of brain anatomy were acquired from the same group of participants, and wholebrain voxel-based morphometry (VBM) analyses (Ashburner and Friston, 2000; Ashburner, 2007) were applied to investigate whether gray matter volume elsewhere in the brain covaried with that of retinotopic visual areas. Subsequently, to validate our findings, we performed independent ROI analysis using Freesurfer segmentation (Desikan et al., 2006) and anatomical atlases (Morosan et al., 2001; Maldjian et al., 2003) on the structural MRI images from the same group of participants. Finally, we further explored and replicated our findings using ROI analyses in an independent group of 130 participants (but now without functional retinotopic mapping).

Participants. Functional visual mapping plus structural MRI images were acquired from a group of 30 healthy young adults ( 17 females, 13 males; aged 18-35). Twelve were native English speakers, and although all were residents in United Kingdom, they originated from a wide range of different countries. Their educational attainment ranged from completion of high school to acquisition of a higher research degree $(\mathrm{PhD})$. To further assess their cognitive abilities, we acquired performance IQ measures from a subset of 25 participants.

Structural MRI images alone were acquired from another independent group of 130 healthy young adults (76 females, 54 males; aged 18-39).

All participants had normal or corrected-to-normal vision. Written informed consent was given by all participants, and the study was approved by the local ethics committee.

Data acquisition (30 participants). In the main group of 30 participants, blood oxygenation level-dependent contrast visual mapping and structural MRI images were acquired from each participant using a Siemens Trio 3 T MRI scanner with a 32-channel headcoil [visual mapping: echo-planar imaging sequence, TR, $3.06 \mathrm{~s}$; echo spacing, $0.56 \mathrm{~ms}$; matrix size, $96 \times 96$; resolution, $2.3 \times 2.3 \times 2 \mathrm{~mm}$; MRI image: T1-weighted modified driven equilibrium Fourier transform (MDEFT) sequence, TR, $7.92 \mathrm{~ms}$; TE, $2.48 \mathrm{~ms}$; flip angle, $16^{\circ}$; field of view, $256 \times 240$; 176 slices; resolution, $1 \times 1 \times 1 \mathrm{~mm}$ ].

To map visual areas (Sereno et al., 1995), retinotopic mapping stimuli were presented on a screen in the back of the scanner and viewed through a mirror on the headcoil (viewing distance, $72 \mathrm{~cm}$ ). Stimuli were highcontrast flickered checkerboards (size, up to 16 visual degrees; flicker rate, $4 \mathrm{~Hz}$ ) on a gray background. In the polar mapping scan, stimuli were a wedge pattern (radius, 8 visual degrees) rotating smoothly in clockwise or anticlockwise direction around a small fixation cross for 10 cycles at the speed of $61.2 \mathrm{~s} / \mathrm{cycle}$. In the eccentricity mapping scan, stimuli were a ring pattern (maximal width, 3 visual degrees) contracting smoothly in polar direction around a small fixation cross for 15 cycles at the speed of $45.9 \mathrm{~s} / \mathrm{cycle}$. To keep participants attended to the retinotopic mapping stimuli, at random temporal intervals the checkerboard stimuli would undergo a small pattern shift for $200 \mathrm{~ms}$, and participants were asked to indicate whenever this happened with a button press.

Data acquisition (130 participants). In a separate group of 130 participants, structural MRI images were acquired from each participant using a Siemens Sonata 1.5 T MRI scanner with a single-channel headcoil (T1-weighted MDEFT sequence: TR, $12.24 \mathrm{~ms}$; TE, $3.56 \mathrm{~ms}$; flip angle, $23^{\circ}$; field of view, $256 \times 256$; 176 slices; resolution, $1 \times 1 \times 1 \mathrm{~mm}$ ).
Data analysis: retinotopic mapping. $\mathrm{PMRI}$ data acquired during retinotopic mapping were preprocessed in statistical parametric mapping software (SPM8) implemented in MATLAB (Mathworks), by applying slice time correction, realignment, unwarping, and coregistration to MRI structural data. Fast Fourier transform was applied to fMRI time series to extract the phase and power at stimulation frequency $(10$ cycles/scan for polar mapping; 15 cycles/scan for eccentricity mapping). An $F$ statistic indicating the significance of visual response was calculated by dividing the power at stimulation frequency with the average power across all frequencies. The resulting phase maps were displayed on reconstructed, inflated cortical surfaces using Freesurfer (Fischl et al., 1999).

The dorsal and ventral boundaries of the visual areas (V1, V2, V3) were delineated manually according to mirror reversals in the polar map. The inner and outer edges of visual areas were defined by thresholding the visual responses in the polar map at the significance level of $p<0.05$ and were confirmed with the eccentricity map. The gray matter volume of dorsal and ventral parts of each region (i.e., V1d, V1v, V2d, V2v, V3d, $\mathrm{V} 3 \mathrm{v}$ ) was calculated by summing up the volume values of all vertices within that part.

Data analysis: whole-brain voxel-based morphometry analysis. VBM is a whole-brain, unbiased, semiautomated technique that characterizes focal volumetric differences in brain structure using a general linear model (Ashburner and Friston, 2000). MRI structural images were preprocessed in SPM8 using VBM DARTEL (diffeomorphic anatomical registration through exponentiated lie algebra) algorithm (Ashburner, 2007). First, structural image from each participant was segmented into different tissues (gray matter, white matter), corrected for image intensity nonuniformity caused by gradient distortions, normalized to a standard T1-weighted template, in a recursive manner. Next, the gray matter segment images from all participants were affine aligned and iteratively matched to a template generated from their own mean. To ensure that the total amount of gray matter was conserved before and after spatial transformation, the transformed images were rescaled, on a voxel basis, by the Jacobian determinants of the deformations. Finally, the gray matter images were smoothed with a Gaussian kernel (full-width at halfmaximum, $8 \mathrm{~mm}$ ) and affine registered to Montreal Neurological Institute (MNI) stereotactic space. These preprocessing procedures allow the gross morphological differences across participants to be removed without affecting the regional gray matter volumes.

Statistical analysis was performed on preprocessed gray matter images using general linear model as implemented in SPM8. We searched for brain regions that positively or negatively covaried with the volume of V1, V1d, V1v, V2, V3, or the whole brain. We conducted regression analysis with the gray matter volume of each region representing the regressor of interest in the design matrix. The gender and age of participants were also included in the design matrix as two regressors of no interest to regress out any gender-related or age-related effects. $T$ statistic maps reflecting the correlation between each regressor and regional gray matter volume were created and thresholded at $p<0.001$ to localize significant clusters. Statistical inferences were based on $p<0.05$ familywise error corrected (nonstationary cluster-level correction) for multiple comparisons across the whole brain (Hayasaka et al., 2004).

When VBM analysis was used to characterize cortical regions that showed positive or negative correlations with visual areas $(\mathrm{V} 1, \mathrm{~V} 1 \mathrm{~d}, \mathrm{~V} 1 \mathrm{v}$, $\mathrm{V} 2, \mathrm{~V} 3)$, the interindividual difference in overall brain volume was controlled by proportional scaling, in which the volume of each voxel was scaled by an individual's total brain gray matter volume (the scaling was applied to visual areas as well as to preprocessed gray matter images). Proportional scaling was chosen over an ANCOVA approach (in which the total gray matter volume was entered as a regressor of no interest in the design matrix) due to the existence of correlation between the gray matter volume of visual area and the gray matter volume of whole brain $(\mathrm{V} 1, r=-0.36, p<0.05 ; \mathrm{V} 2, r=-0.26, p=0.17$; V3, $r=0.31, p=0.10$; $\mathrm{V} 1 \mathrm{~d}, r=-0.30, p=0.10 ; \mathrm{V} 1 \mathrm{v}, r=-0.26, p=0.17)$. If two regressors are correlated, the general linear model cannot distinguish variance attributable uniquely to one or other regressor that may lead to ambiguous results (Andrade et al., 1999). Details of the VBM results using proportional scaling are listed in Table 1. For comparison, VBM results using ANCOVA are listed in Table 2. Clearly, results were highly consistent between two approaches, but 
Table 1. Detailed VBM results using proportional scaling to control for interindividual differences in whole brain volume

\begin{tabular}{|c|c|c|c|c|c|c|}
\hline Regressor & Cluster location and laterality & & $\begin{array}{l}\text { Peak voxel MNI } \\
\text { coordinates }\end{array}$ & $\begin{array}{l}\text { Number of voxels } \\
\text { (resels) in cluster }\end{array}$ & $\begin{array}{l}\text { Cluster-level } p \text { value } \\
\text { with FWE correction }\end{array}$ & $\begin{array}{l}\text { Peak voxel } \\
\text { tscore }\end{array}$ \\
\hline \multirow[t]{2}{*}{ V1 positive } & Primary auditory cortex (BA41/42) & $\mathbf{R}$ & $(66,-12,13)$ & $419(1.29)$ & 0.025 & 5.43 \\
\hline & Superior parietal lobule (BA5/7) & $\mathrm{R}$ & $(22,-61,31)$ & $66(0.37)$ & 0.339 & 4.35 \\
\hline \multirow[t]{8}{*}{ V1 negative } & Anterior prefrontal cortex, ventromedial part (BA10/11) & $\mathrm{L} / \mathrm{R}$ & $(9,74,6)$ & $3314(6.2)$ & $<10^{-4}$ & 6.11 \\
\hline & Anterior prefrontal cortex, dorsolateral part (BA10/46) & $\mathrm{L} / \mathrm{R}$ & $(42,44,10)$ & $346(1.01)$ & 0.048 & 4.69 \\
\hline & & & $(-48,30,16)$ & $147(0.46)$ & 0.243 & 4.05 \\
\hline & & & $(30,68,10)$ & $34(0.18)$ & 0.688 & 4.42 \\
\hline & & & $(50,30,21)$ & $23(0.08)$ & 0.928 & 3.80 \\
\hline & Middle occipital gyrus (BA18) & $\mathrm{R}$ & $(33,-88,-3)$ & $200(0.94)$ & 0.058 & 5.49 \\
\hline & Anterior prefrontal cortex, dorsomedial part (BA10/9) & $\mathrm{R}$ & $(26,65,25)$ & $132(0.49)$ & 0.224 & 5.31 \\
\hline & & & $(15,60,37)$ & $75(0.17)$ & 0.720 & 4.80 \\
\hline V1d positive & Cerebellum & $\mathrm{L}$ & $(-39,-64,-48)$ & $117(0.21)$ & 0.617 & 3.86 \\
\hline \multirow[t]{3}{*}{ V1d negative } & Anterior prefrontal cortex, dorsolateral part (BA10/46) & $\mathbf{L} / \mathbf{R}$ & $(-42,39,16)$ & $487(1.38)$ & 0.020 & 4.90 \\
\hline & & & $(44,44,12)$ & $239(0.87)$ & 0.068 & 4.81 \\
\hline & & & $(32,60,25)$ & $24(0.10)$ & 0.884 & 3.84 \\
\hline \multirow[t]{5}{*}{ V1v positive } & Primary auditory cortex (BA41/42) & $\mathrm{R}$ & $(68,-13,13)$ & $321(0.91)$ & 0.061 & 5.03 \\
\hline & Primary motor cortex (BA4) & $\mathrm{L}$ & $(-64,-18,21)$ & $229(0.62)$ & 0.147 & 4.95 \\
\hline & Superior parietal lobule (BA5/7) & $\mathrm{R}$ & $(22,-61,31)$ & $152(0.60)$ & 0.157 & 5.08 \\
\hline & Inferior temporal cortex (BA20) & $\mathrm{L} / \mathrm{R}$ & $(-34,-15,-32)$ & $61(0.28)$ & 0.475 & 4.56 \\
\hline & & & $(54,-18,-20)$ & $25(0.14)$ & 0.784 & 3.84 \\
\hline \multirow[t]{4}{*}{ V1v negative } & Anterior prefrontal cortex, ventromedial part (BA10/11) & $\mathrm{L} / \mathrm{R}$ & $(8,64,4)$ & $2558(4.3)$ & $<10^{-4}$ & 6.05 \\
\hline & & & $(-10,57,10)$ & $73(0.16)$ & 0.737 & 4.78 \\
\hline & Anterior prefrontal cortex, dorsomedial part (BA10/9) & $\mathrm{R}$ & $(22,66,25)$ & $125(0.68)$ & 0.119 & 4.87 \\
\hline & Middle occipital gyrus (BA18) & $\mathrm{R}$ & $(33,-88,-3)$ & $88(0.36)$ & 0.357 & 4.85 \\
\hline \multirow[t]{4}{*}{ V2 positive } & Superior temporal cortex (BA22) & $\mathrm{L}$ & $(-57,-10,7)$ & $181(0.82)$ & 0.078 & 5.39 \\
\hline & Cerebellum & $\mathrm{L} / \mathrm{R}$ & $(-26,-45,-39)$ & $127(0.27)$ & 0.494 & 4.31 \\
\hline & & & $(16,-63,-41)$ & $63(0.06)$ & 0.957 & 4.17 \\
\hline & & & $(14,-45,-41)$ & $15(0.05)$ & 0.966 & 4.04 \\
\hline \multirow[t]{4}{*}{ V2 negative } & Posterior cingulated cortex (BA31) & $\mathrm{R}$ & $(24,-78,24)$ & $173(0.62)$ & 0.143 & 3.95 \\
\hline & Broca's area (BA44/45) & $\mathrm{L}$ & $(-48,27,18)$ & $35(0.26)$ & 0.515 & 4.40 \\
\hline & Superior parietal lobule (BA5/7) & $\mathrm{R}$ & $(8,-63,42)$ & $17(0.16)$ & 0.737 & 4.10 \\
\hline & Supplementary motor area (BA8) & $\mathrm{R}$ & $(22,23,51)$ & $20(0.08)$ & 0.924 & 3.67 \\
\hline V3 positive & Inferior temporal cortex (BA20) & $\mathrm{R}$ & $(48,-20,-33)$ & $83(0.19)$ & 0.676 & 4.25 \\
\hline \multirow[t]{3}{*}{ V3 negative } & Middle occipital gyrus (BA18) & $\mathrm{R}$ & $(34,-82,-5)$ & $43(0.19)$ & 0.675 & 4.09 \\
\hline & Cerebellum & $\mathrm{L}$ & $(-39,-27,-9)$ & $35(0.16)$ & 0.743 & 4.33 \\
\hline & Posterior cingulated cortex (BA30) & $\mathrm{L}$ & $(-21,-51,12)$ & $42(0.09)$ & 0.897 & 3.71 \\
\hline
\end{tabular}

For completeness, clusters that survive a height threshold $p<0.001$ (uncorrected) and an extent threshold of 10 voxels ( 0.05 resels) are reported. Clusters that survive whole-brain corrected $p<0.05$ (FWE nonstationary cluster-leve correction) are indicated in bold.

the statistical power was weaker in the ANCOVA approach due to the multicollinearity between regressors. These analyses were applied to the main group of 30 participants.

When VBM was used to characterize cortical regions that showed the strongest correlation with the gray matter volume of the brain, the volume of each voxel in the preprocessed gray matter image was scaled by an individual's whole-brain volume. This allowed us to localize regions that not only scaled with the brain but also increased in relative size with the brain. Since this analysis requires no retinotopic mapping, it was applied to the main group of 30 participants and validated in an independent data set of 130 participants.

Data analysis: follow-up ROI analyses. To validate and further explore the findings from the whole-brain VBM analyses above, we conducted additional follow-up and confirmatory analyses using regions of interest. In particular, we explored whether the anatomical relationship between the gray matter volume of V1 and the gray matter volume of other brain region reported by our VBM result is driven by cortical thickness or by cortical surface area. For each brain region identified from significant clusters in VBM results, a corresponding ROI was selected according to operator-independent Freesurfer segmentation (Desikan et al., 2006). Subsequently, the surface area or average cortical thickness of this ROI was subtracted and plotted against the surface area or average cortical thickness of V1. This analysis was applied to the main group of 30 participants.

We also explored whether our finding on V1 and its relationship with other brain region can be generalized to other primary sensory cortex such as primary auditory cortex (A1). As there is no established func- tional MRI protocol for delineating the borders of primary auditory cortex from the surrounding regions, we used a widely accepted probabilistic atlas (Te1.0) (Morosan et al., 2001), or Freesurfer operatorindependent segmentation (transverse temporal cortex) (Desikan et al., 2006), to define A1. For each of these two ways of defining Al, we calculated the correlation coefficient between the gray matter volume of A1 and that of another brain region defined in the corresponding way, anatomical atlas (Maldjian et al., 2003) or Freesurfer segmentation (Desikan et al., 2006). Since this analysis requires no retinotopic mapping, it was applied to the main group of 30 participants and validated in an independent data set of 130 participants.

In our ROI analysis, the individual differences in overall brain size were accounted for by proportional scaling: the correlation between two regional volumes (surface areas, thicknesses) was calculated after normalizing it relative to the whole-brain volume (surface area, thickness).

\section{Results}

Retinotopic mapping analysis (30 participants)

Across the 30 participants studied, we found large interindividual variability in the gray matter volume of functionally defined visual cortex (V1, 4824-8933 $\mathrm{mm}^{3}$; V2, 4061-8591 $\mathrm{mm}^{3}$; V3, $4159-9034 \mathrm{~mm}^{3}$ ) and considerable variability in volume of the whole brain $\left(392,750-674,120 \mathrm{~mm}^{3}\right)$. This degree of variability (whole brain, 40\%; visual cortex, twofold) is consistent with previous reports (Stensaas et al., 1974; Dekaban, 1978; Dougherty et 
Table 2. Detailed VBM results using ANCOVA to control for interindividual differences in whole brain volume

\begin{tabular}{|c|c|c|c|c|c|c|}
\hline Regressor & Cluster location and laterality & & $\begin{array}{l}\text { Peak voxel MNI } \\
\text { coordinates }\end{array}$ & $\begin{array}{l}\text { Number of voxels } \\
\text { (resels) in cluster }\end{array}$ & $\begin{array}{l}\text { Cluster-level } p \text { value } \\
\text { with FWE correction }\end{array}$ & $\begin{array}{l}\text { Peak voxel } \\
t \text { score }\end{array}$ \\
\hline \multirow[t]{3}{*}{ V1 positive } & Primary auditory cortex (BA41/42) & $\mathrm{R}$ & $(62,-10,13)$ & $542(1.73)$ & 0.009 & 5.15 \\
\hline & Superior parietal lobule (BA5/7) & $\mathrm{R}$ & $(22,-61,33)$ & $60(0.29)$ & 0.439 & 4.59 \\
\hline & & & $(30,-48,74)$ & $53(0.20)$ & 0.626 & 4.51 \\
\hline \multirow[t]{7}{*}{ V1 negative } & Anterior prefrontal cortex (BA10) & $\mathrm{L} / \mathrm{R}$ & $(-32,47,-2)$ & $27(0.25)$ & 0.517 & 3.99 \\
\hline & & & $(38,42,3)$ & $74(0.20)$ & 0.626 & 3.96 \\
\hline & & & $(-30,68,1)$ & $22(0.11)$ & 0.829 & 3.86 \\
\hline & Middle occipital gyrus (BA18) & $L / R$ & $(14,-82,25)$ & $46(0.36)$ & 0.333 & 4.54 \\
\hline & & & $(-20,-87,15)$ & $17(0.15)$ & 0.735 & 4.13 \\
\hline & & & $(36,-84,-6)$ & $23(0.11)$ & 0.844 & 3.81 \\
\hline & Orbitofrontal cortex (BA11/47) & $\mathrm{R}$ & $(32,34,-26)$ & $29(0.08)$ & 0.919 & 4.00 \\
\hline \multirow[t]{2}{*}{ V1d positive } & Superior parietal lobule (BA5/7) & $L / R$ & $(-27,-69,58)$ & $282(1.37)$ & 0.019 & 5.36 \\
\hline & & & $(33,-48,72)$ & $22(0.16)$ & 0.711 & 4.42 \\
\hline \multirow[t]{2}{*}{ V1d negative } & Anterior prefrontal cortex, dorsolateral part (BA10/46) & $L / R$ & $(45,44,10)$ & $10(0.10)$ & 0.871 & 3.77 \\
\hline & & & $(-42,39,16)$ & $17(0.07)$ & 0.925 & 3.65 \\
\hline \multirow[t]{3}{*}{ V1v positive } & Primary auditory cortex (BA41/42) & $L / R$ & $(66,-12,12)$ & $70(0.17)$ & 0.690 & 3.92 \\
\hline & & & $(-57,-27,10)$ & $13(0.07)$ & 0.926 & 3.84 \\
\hline & Superior parietal lobule (BA5/7) & $\mathrm{R}$ & $(22,-61,33)$ & $54(0.32)$ & 0.388 & 4.66 \\
\hline \multirow[t]{8}{*}{ V1v negative } & Anterior prefrontal cortex, ventromedial part (BA10) & $L / R$ & $(6,64,6)$ & $242(0.58)$ & 0.155 & 4.89 \\
\hline & & & $(-36,54,1)$ & $23(0.13)$ & 0.791 & 3.74 \\
\hline & & & $(-34,66,1)$ & $32(0.12)$ & 0.806 & 3.90 \\
\hline & Middle occipital gyrus (BA18) & $L / R$ & $(12,-82,25)$ & $23(0.26)$ & 0.497 & 4.14 \\
\hline & & & $(-14,-88,-20)$ & $58(0.18)$ & 0.680 & 4.00 \\
\hline & Anterior prefrontal cortex, dorsomedial part (BA10/9) & $\mathrm{R}$ & $(15,60,37)$ & $37(0.14)$ & 0.758 & 4.59 \\
\hline & Angular gyrus (BA39) & $\mathrm{L}$ & $(-32,-63,39)$ & $14(0.16)$ & 0.716 & 3.95 \\
\hline & Cerebellum & $\mathrm{L}$ & $(-28,-39,-41)$ & $28(0.11)$ & 0.853 & 3.91 \\
\hline V2 positive & No suprathreshold clusters & & & & & \\
\hline \multirow[t]{11}{*}{ V2 negative } & Hippocampal gyrus & $L / R$ & $(-21,0,-20)$ & $1641(2.7)$ & 0.002 & 4.84 \\
\hline & & & $(14,-12,-33)$ & $1915(2.3)$ & 0.004 & 5.03 \\
\hline & & & $(4,-37,6)$ & $34(0.06)$ & 0.944 & 3.95 \\
\hline & Broca's area (BA44/45) & $\mathrm{L}$ & $(-45,6,7)$ & $52(0.41)$ & 0.029 & 4.71 \\
\hline & & & $(-46,24,15)$ & $39(0.32)$ & 0.398 & 4.10 \\
\hline & Inferior occipital gyrus (BA19) & $\mathrm{R}$ & $(15,-84,27)$ & $278(1.18)$ & 0.030 & 4.28 \\
\hline & Orbitofrontal cortex (BA11/47) & $L / R$ & $(-16,29,-18)$ & $426(1.02)$ & 0.044 & 4.33 \\
\hline & & & $(20,36,-29)$ & $309(0.57)$ & 0.161 & 4.99 \\
\hline & Middle temporal cortex (BA37) & $\mathrm{L}$ & $(-57,-55,-11)$ & $156(0.28)$ & 0.466 & 4.42 \\
\hline & Thalamus & $\mathrm{R}$ & $(16,-27,15)$ & $158(0.21)$ & 0.594 & 4.13 \\
\hline & Posterior cingulated gyrus (BA23) & $\mathrm{L}$ & $(-6,-36,25)$ & $126(0.09)$ & 0.884 & 4.01 \\
\hline V3 positive & No suprathreshold clusters & & & & & \\
\hline \multirow[t]{6}{*}{ V3 negative } & Middle occipital gyrus (BA18) & $\mathrm{R}$ & $(33,-82,-5)$ & $140(0.70)$ & 0.106 & 4.69 \\
\hline & Cerebellum & $\mathrm{R}$ & $(14,-33,-17)$ & $19(0.24)$ & 0.536 & 3.93 \\
\hline & & & $(6,-49,1)$ & $16(0.15)$ & 0.754 & 3.96 \\
\hline & & & $(9,-39,-32)$ & $59(0.07)$ & 0.937 & 4.20 \\
\hline & Orbitofrontal cortex (BA11/47) & $\mathrm{R}$ & $(38,20,-8)$ & $24(0.23)$ & 0.567 & 4.10 \\
\hline & Anterior cingulate gyrus (BA32) & $\mathrm{L}$ & $(-2,36,22)$ & $14(0.09)$ & 0.900 & 3.83 \\
\hline
\end{tabular}

For completeness, clusters that survive a height threshold $p<0.001$ (uncorrected) and an extent threshold of 10 voxels ( 0.05 resels) are reported.

al., 2003) but now in a larger sample acquired noninvasively through functional neuroimaging.

One striking property of early visual cortex is its spatial arrangement. Due to the retinotopic organization of the early visual system, the upper and lower visual fields are represented by the ventral and dorsal portions, respectively, of areas V1-V3 (Wandell et al., 2007). Because the foveal confluence is spared by the functional mapping procedure, the representations of the upper and lower visual fields in V2/V3 are separated from each other by V1 (Fig. 1A). The two parts also differ with regard to their processing capacity; for example, illusory contour processing is enhanced in the lower compared with the upper visual field (Rubin et al., 1996). We therefore investigated whether dorsal V1 (representing the lower visual field) and ventral V1 (representing the upper visual field) showed separate structural interrelationships with other retinotopic visual cortices (V2, V3).
We performed a common factor analysis (with orthogonal rotation) on the gray matter volume of early visual cortices (V1d, V1v, V2d, V2v, V3d, and V3v) and investigated how many common factors served to accurately characterize their interindividual variability. In contrast to principal component analysis that simply summarizes the data with fewer dimensions, common factor analysis allowed us to find the hidden variables (i.e., factors) that affected the pattern of relationships in our data and understand the nature of these hidden variables (Velicer and Jackson, 1990). Our analysis suggested that one common factor failed to explain all the variability presented in the data $(p<0.05$; reject the null hypothesis of one common factor), but two common factors were sufficient $(p=0.24$; failure to reject the null hypothesis of two common factors). Importantly, the two common factors separated the dorsal and the ventral areas. Specifically, we found a joint covariation among V1d, V2d, V3d (Fig. 

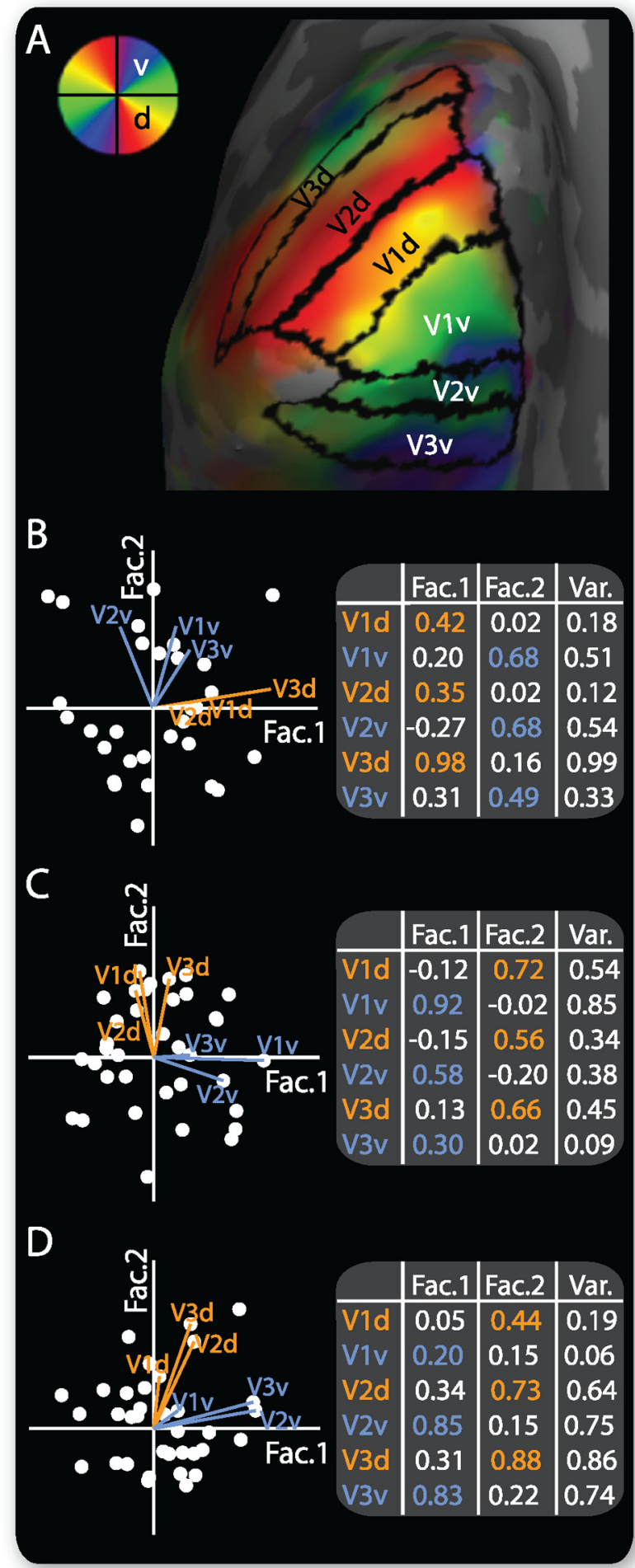

Figure 1. Separation between dorsal V1 and ventral V1 in anatomical covariance with associated visual cortices. $\boldsymbol{A}$, Retinotopic map sample from a representative participant illustrates the dorsal and ventral halves of early retinotopic visual cortices (V1d, V1v, V2d, V2v, V3d, V3v). To map the visual areas, neuronal responses (measured by fMRI) to stimulation in different visual fields (see color-wheel legend) were projected onto an inflated cortical surface, and the boundaries of the visual areas were delineated manually by the mirror reversals in the retinotopic map. $\boldsymbol{B}-\boldsymbol{D}$, Results of common factor analysis on gray matter volume $(\boldsymbol{B})$, surface area $(\boldsymbol{C})$, and cortical thickness ( D) of early retinotopic visual cortices (V1d, V1v, V2d, V2v, V3d, V3v) were visualized using the biplot figure (left panel) and the statistic table (right panel), indicating that the interindividual variability in six visual cortices can be characterized with two common factors $(N=30)$. The values of the factor loadings (the correlation coefficients between the visual cortices and the underlying factor) and the communality (the percentage of variance explained
$1 B$, factor 1 ), and among V1v, V2v, V3v (Fig. $1 B$, factor 2). Moreover, the same covariation patterns among V1d, V2d, V3d, and among V1v, V2v, V3v were found both in surface area (Fig. 1C) and in cortical thickness (Fig. 1D). These results suggest that a dorsal-ventral segregation of functionally defined retinotopic anatomy was present as early as V1, and this segregation is controlled by cortical surface area and cortical thickness separately.

\section{Whole-brain voxel-based morphometry analyses (30 participants)}

Using V1 gray matter volume (determined in the retinotopic analyses above) of each participant as the regressor of interest in a VBM analysis, we found that the gray matter volume of V1 covaried positively with the gray matter volume of anatomically defined primary auditory cortex (A1) (Fig. $2 \mathrm{~A}$, Table 1 ) [peak voxel coordinates: $(64,-12,13)$, BA42/Te1.0; $T$ score, $5.43 ; p<0.05$, corrected] (Morosan et al., 2001). In contrast, anterior prefrontal cortex (aPFC) showed a negative correlation with the gray matter volume of V1 (Fig. 2A, Table 1) [peak voxel coordinates: $(9,74,6)$, BA10; $T$ score $=6.11 ; p<0.0001$, corrected $]$.

To test whether these anatomical covariances were specific to V1 or associated with retinotopic visual cortices more generally, we also conducted independent whole-brain VBM analyses searching for regions whose gray matter volume correlates with the gray matter volume of V2 or V3. However, no such region was found for either positive or negative correlations (Table 1) $(p>$ 0.05 , corrected). Thus, the positive and negative correlations with gray matter volume of $\mathrm{A} 1$ and $\mathrm{aPFC}$, respectively, were specific to the gray matter volume of primary visual cortex and did not arise from the large degree of interindividual variability in the volume of visual cortex or the occipital lobe per se.

Our findings from the retinotopic mapping analysis described above suggested that a dorsal-ventral segregation was present as early as V1, even though the dorsal and ventral portions of V1 are anatomically adjacent. We therefore further investigated how the volume of V1d and V1V related to the rest of the cortex by conducting separate VBM analysis using the volumes of V1d and V1v as regressor of interest, respectively. We found a clear dissociation between V1d and V1v in their relationship with aPFC. While V1d showed a focal negative correlation with the dorsolateral part of aPFC (Fig. 2 B, Table 1) [peak voxel coordinates: $(-42,39$, 16), BA10/46; T score, 4.90; $p<0.05$, corrected], V1v correlated negatively with the ventromedial part (Fig. 2C, Table 1) [peak voxel coordinates: $(8,64,4)$, BA10; $T$ score, $6.05 ; p<0.0001$, corrected].

\section{Follow-up region-of-interest analyses}

Roles of cortical thickness and surface area in V1-A1, V1-aPFC relationships (30 participants)

The correlations between V1 gray matter volume and the gray matter volume of A1 or aPFC that we observed in whole-brain VBM analyses could be driven by either cortical surface area or cortical thickness, or some combination of the two. In follow-up analyses, we explored this possibility. We used Freesurfer to perform an operator-independent segmentation of auditory cortex (specifically, transverse temporal cortex) (Desikan et al., 2006) and aPFC (specifically, rostral middle frontal cortex) in our 30 participants. We then compared how interindividual variability in cor-

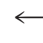

by the two common factors) are listed in the table and can be inspected from the direction and length of the vectors in the biplot figure. 


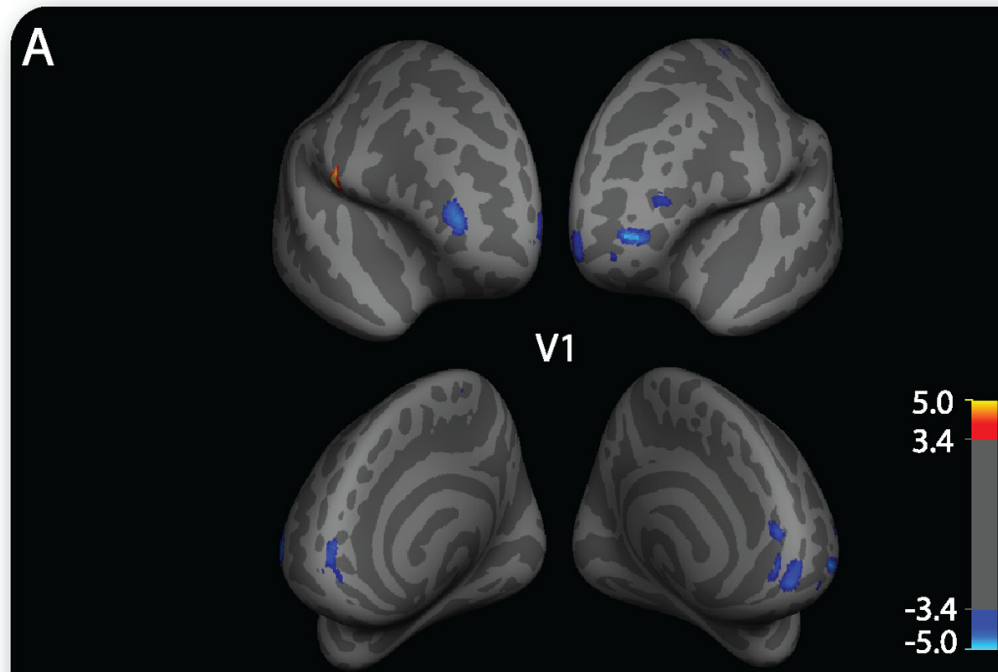

B
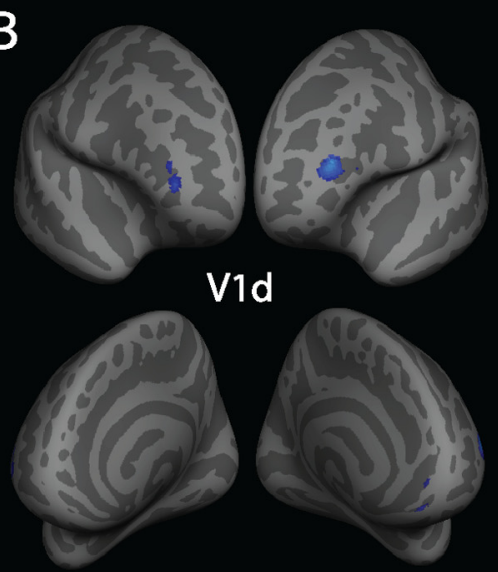

C

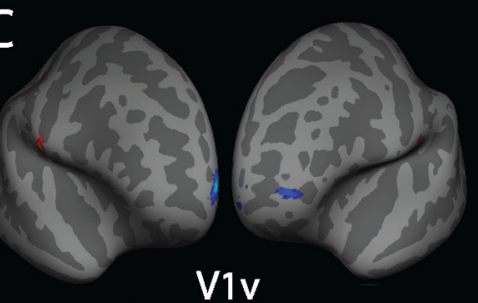

D
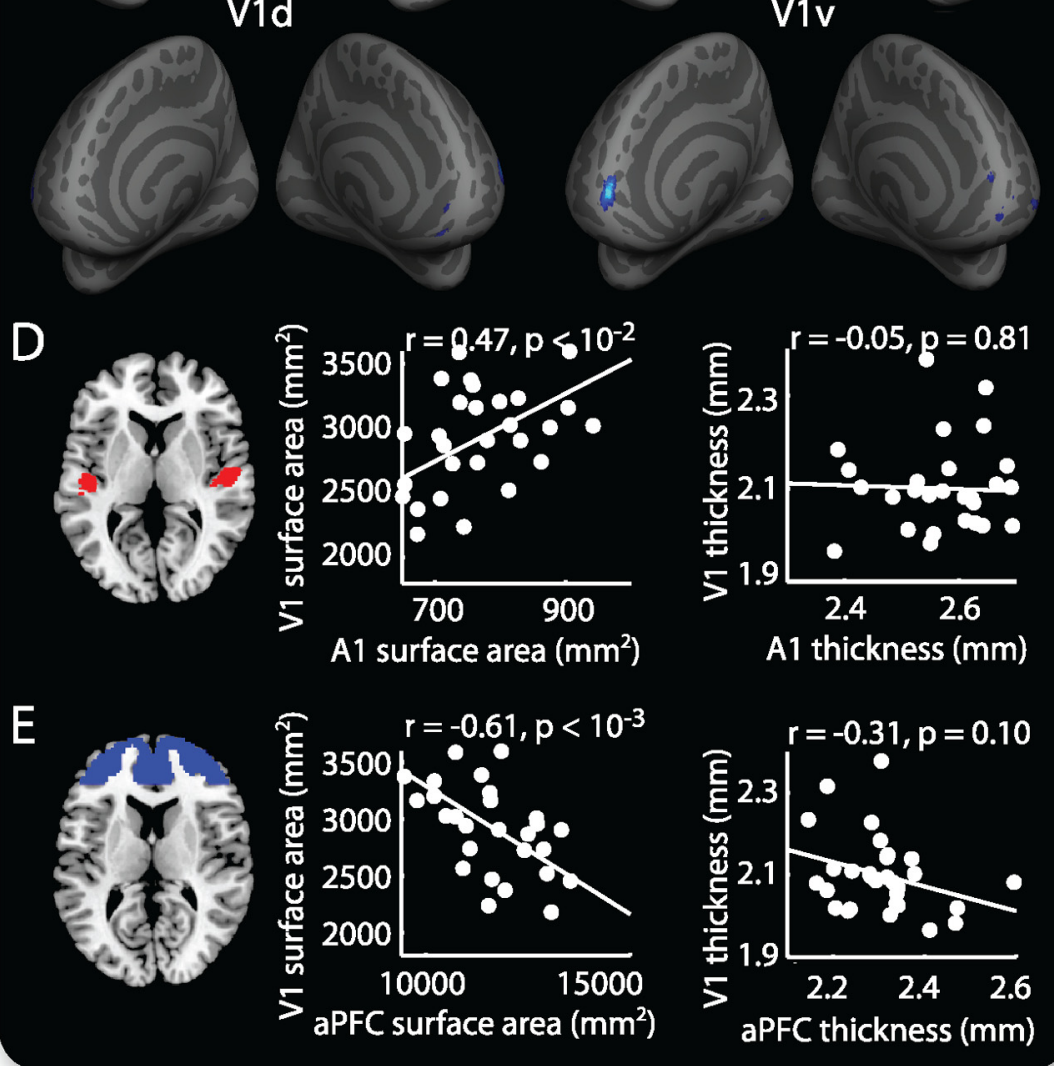

Figure 2. Anatomical relationship between primary visual cortex and the rest of the brain. $\boldsymbol{A}$, Statistical $(T$ ) maps for positive (hot color: red, yellow) and negative (cold color: blue, cyan) correlations with V1 in gray matter volume were overlaid on an inflated cortical surface (thresholded at $T>3.4$ fordisplay purposes). Significant clusters ( $p<0.05$, nonstationary cluster-level correction) werefound in A1 (positivecorrelation, red-yellow clusters) and aPFC (negative correlation, blue-cyan clusters) $(N=30) . \boldsymbol{B}, \boldsymbol{C}$, Statistical ( $T$ ) maps for positive (hot color: red, yellow) and negative (cold color: blue, cyan) correlations with V1d $(\boldsymbol{B})$ or V1v $(\boldsymbol{C}$ in gray matter volume were overlaid on an inflated cortical surface (thresholded at $T>3.4 \mathrm{for}$ display purposes). Significantclusters ( $p<0.05$, nonstationary cluster-level correction) werefound in dorsolateral aPFC fornegative correlation with V1d, and in ventromedial aPFC for negative correlation with V1v $(N=30) \cdot \boldsymbol{D}, \boldsymbol{E}$, Surface area and cortical thickness of V1 were plotted against that of $\mathrm{A} 1(\boldsymbol{D})$ and aPFC $(\boldsymbol{E})$, illustrating that the positive correlation between V1 and A1 volume, as well as the negative correlation between V1 and aPFC volume, were driven by cortical surface area instead of cortical thickness $(N=30)$. tical thickness and surface area derived from these segmented ROIs related to V1 surface area and thickness measured through the retinotopic mapping.

Figure $2 D$ shows these relationships for auditory cortex. While interindividual variability in the surface areas of primary auditory and primary visual cortex were strongly and significantly correlated $(r=$ $0.47 ; p<0.01 ; N=30$ ), there was no significant correlation between cortical thickness of the two areas $(r=-0.05 ; p=$ $0.81 ; N=30)$. Figure $2 E$ shows these relationships for aPFC. Again, interindividual variability in the surface areas of aPFC and primary visual cortex were strongly and negatively correlated $(r=$ $-0.61 ; p<0.001 ; N=30$ ), but there was no significant correlation between cortical thickness of these two areas $(r=$ $-0.31 ; p=0.10 ; N=30)$.

Negative correlation between $A 1$ and aPFC (30 participants and replication in 130 participants)

Our whole-brain VBM analysis showed that the gray matter volume of primary visual cortex correlated positively with the gray matter volume of primary auditory cortex but negatively with that of anterior prefrontal cortex. In contrast to sensory cortices, anterior prefrontal cortex is the phylogenetically newest cortical structure and supports higher-order cognitive functions in humans (Ramnani and Owen, 2004). The negative correlation we observed between aPFC and V1 hints at a reciprocal anatomical relationship between aPFC and primary sensory cortices in general.

To test this hypothesis, we conducted ROI analysis examining the correlation between aPFC and A1 in the first group of 30 participants as well as an independent group of 130 participants. Unlike primary visual cortex that can be localized accurately on an individual basis using widely accepted retinotopic mapping protocols (Sereno et al., 1995), there is no established functional MRI protocol for delineating the borders of primary auditory cortex from the surrounding regions. Therefore, we defined A1 according to anatomical atlas (Te1.0) (Morosan et al., 2001), or Freesurfer operator-independent segmentation (transverse temporal cortex) (Desikan et al., 2006), and plotted its gray matter volume against the gray matter volume of aPFC defined in the corresponding way, anatomical atlas (BA10) (Maldjian et al., 2003), or Freesurfer segmentation (rostral middle frontal cortex) (Desikan et al., 2006).

We found a strong and highly significant negative correlation between gray 

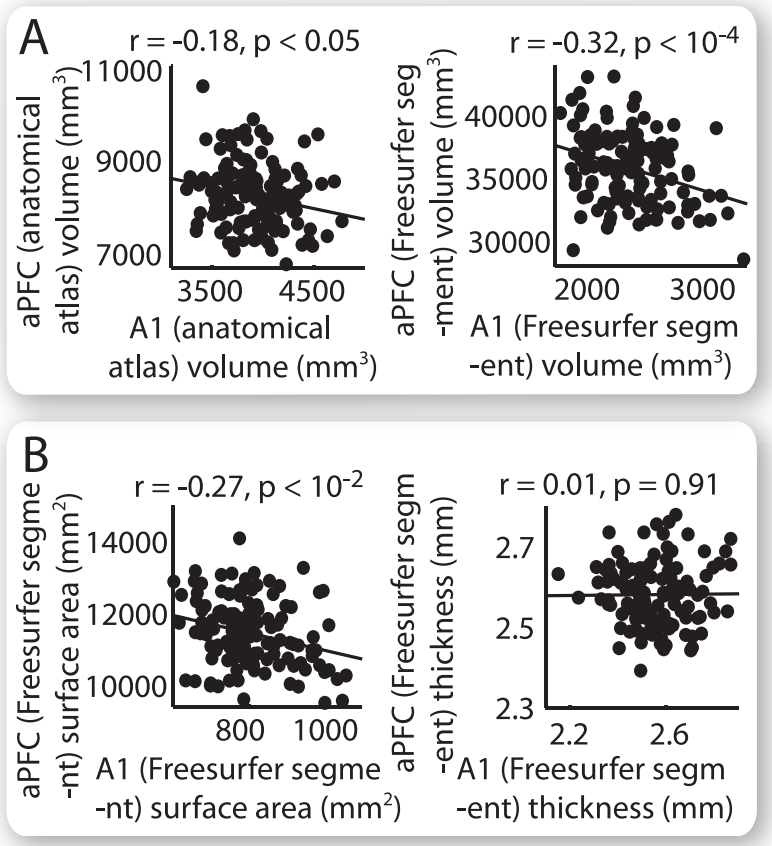

Figure 3. Negative correlation between primary auditory cortex and anterior prefrontal cortex. $\boldsymbol{A}$, Gray matter volume of $\mathrm{A} 1$ was plotted against that of aPFC, illustrating the negative correlation between $\mathrm{A} 1$ and aPFC $(N=130)$. In separate analyses, $\mathrm{A} 1$ and aPFC were defined according to anatomical atlas (Te1.0 and BA10, respectively), or Freesurfer segmentation (transverse temporal cortex and rostral middle frontal cortex, respectively). $\boldsymbol{B}$, Surface area and cortical thickness of A1 were plotted against that of aPFC, illustrating that the negative correlation between $\mathrm{A} 1$ and aPFC volume was driven by cortical surface area instead of cortical thickness $(N=130)$.

matter volume of $\mathrm{A} 1$ and aPFC in the new group of 130 participants (Fig. 3A) (anatomical atlas: $r=-0.18, p<0.05, N=130$; Freesurfer segmentation: $r=-0.32, p<0.0001, N=130$ ) as well as the original group of 30 participants (anatomical atlas: $r=$ $-0.43, p<0.02, N=30$ ). The consistency between the results from two independent groups of participants strengthens our findings. We further explored whether this A1-aPFC anticorrelation is driven by cortical surface area or cortical thickness. We compared how the surface area or thickness of Freesurfer segment A1 (transverse temporal cortex) and aPFC (rostral middle frontal cortex) relate, and found that this negative covariance between aPFC and A1 was driven by cortical surface area rather than cortical thickness (Fig. 3B) (surface area: $r=-0.27, p<$ $0.01, N=130$; thickness: $r=0.01, p=0.91, N=130$ ).

Relationship with variation in whole-brain size (30 participants and 130 participants)

Although overall brain size constrains the size of its components, it does not necessarily follow that there is any shared relationship between them: the size of specific cortical areas need not scale with the overall brain size. Understanding how the volume of a cortical region relates to overall brain volume may help to reveal the ecological role of that cortical area and its associated cognitive functions. For example, as the brain increases in size along hominid evolution (Roth and Dicke, 2005), cortical regions whose size positively covaries with brain size may reflect ecologically important cognitive functions, and the opposite may apply to cortical areas whose size negatively covaries with brain size (Schoenemann, 2006).

We therefore characterized how the gray matter volume of primary visual cortex (measured through retinotopic mapping) and the
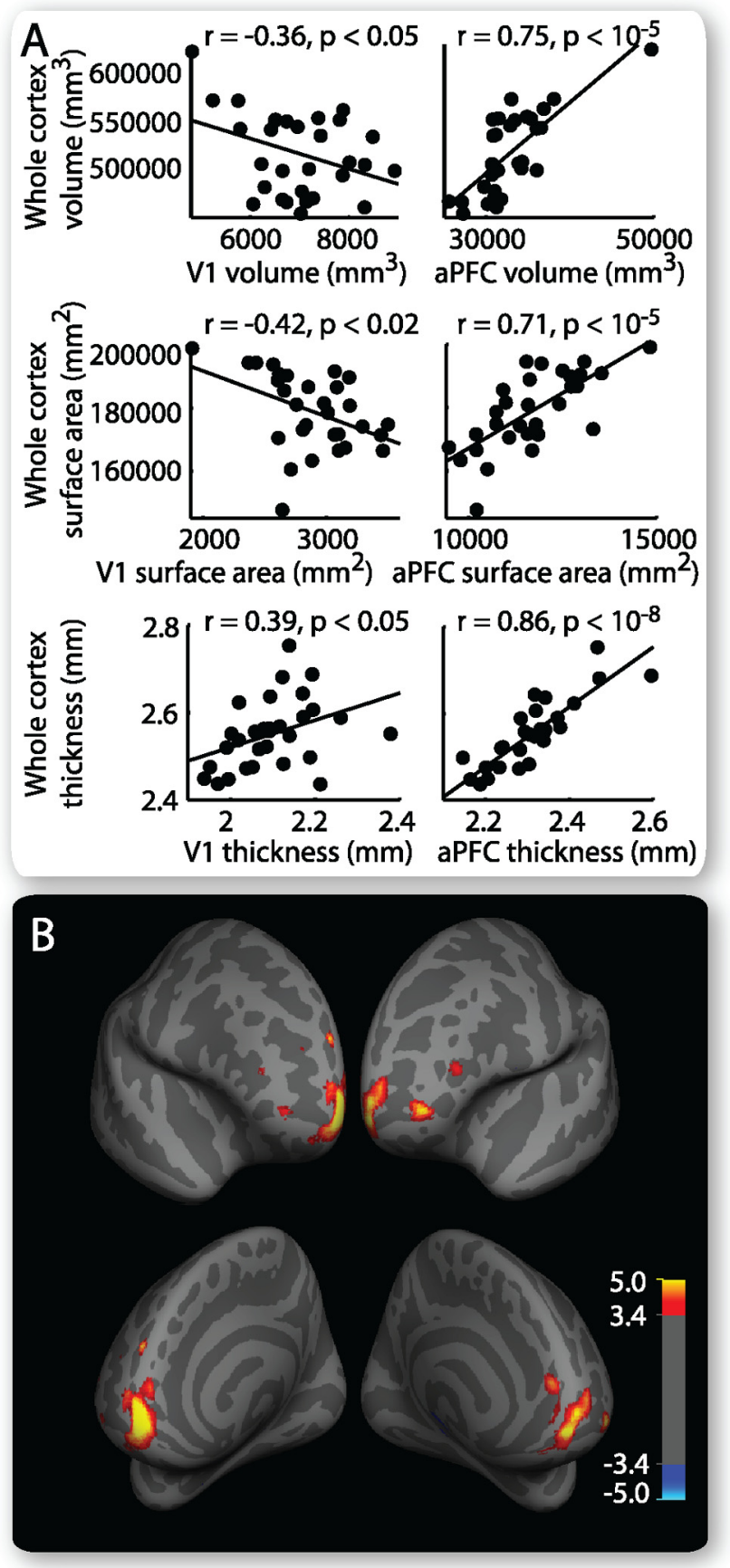

Figure 4. Reciprocal anatomical relationship of primary visual cortex and anterior prefrontal cortex with the whole cortex. $A$, Gray matter volume, surface area, and cortical thickness of the whole cortex were plotted against that of V1 (leftpanel) and that ofaPFC (right panel), indicating an opposite relationship between $\mathrm{V} 1$ and aPFC with the whole cortexin gray mattervolume and surface area $(N=$ 30). $B$, Statistical ( $T$ ) maps for positive (hot color: red, yellow) and negative (cold color: blue, cyan) correlations with the whole cortex in gray matter volume were overlaid on an inflated cortical surface (thresholded at $T>3.4$ for display purposes). A significant cluster ( $p<0.05$, nonstationary clusterlevel correction) of positive correlation was found in $\operatorname{PFC}(N=30)$.

gray matter volume of anterior prefrontal cortex (Freesurfer segmentation rostral middle frontal cortex) related to the volume of the whole cortical sheet. Surprisingly, we found that V1 volume covaried negatively with the whole-brain gray matter volume (Fig. $4 \mathrm{~A}$, left panel) $(r=-0.36 ; p<0.05 ; N=30)$. The same negative correlation held between the surface area of $\mathrm{V} 1$ and that of the whole cortex (Fig. $4 A$, left panel) $(r=-0.42 ; p<0.02 ; N=30)$, while there was a 
positive correlation between $\mathrm{V} 1$ thickness and the whole cortical thickness (Fig. 4A, left panel) $(r=0.39 ; p<0.05 ; N=30)$.

In contrast, aPFC showed a positive correlation with the whole cortex in gray matter volume (Fig. $4 A$, right panel) $(r=0.75 ; p<$ $\left.10^{-5} ; N=30\right)$, surface area (Fig. $4 A$, right panel) $(r=0.71 ; p<$ $\left.10^{-5} ; N=30\right)$, as well as cortical thickness (Fig. $4 A$, right panel) $(r=$ $0.86 ; p<10^{-8} ; N=30$ ). Moreover, a VBM analysis with wholebrain volume as the regressor of interest indicated that, among all cortical areas, aPFC had the strongest positive covariance with the overall brain volume (Fig. $4 B$ ) $[N=30$; peak voxel coordinates: (10, 69, 4), BA10; $T$ score, 7.44; $p<10^{-5}$, corrected]. This VBM result was confirmed and replicated in the second group of 130 participants, which showed again a highly significant correlation between the volume of aPFC and overall brain volume $[N=130$; peak voxel coordinates: $(-22,69,-12)$, BA10; $T$ score, 8.19; $p<10^{-6}$, corrected]. This result hints toward the possibility that aPFC might be ecologically the single most important brain structure and is consistent with the fact that aPFC supports high-order cognitive functions unique or advanced in humans compared with other animals (Ramnani and Owen, 2004; Amodio and Frith, 2006).

\section{Possible confounding factors (30 participants)}

Our VBM analyses included both age and gender as covariates of no interest, so these factors cannot account for our findings. We collected data on performance IQ from 25 of the 30 participants who underwent retinotopic mapping. We did not observe any correlation between IQ and the size of cortical areas determined either through retinotopic mapping (primary visual cortex: $r=0.0403$, $p=0.8481, N=25$ ) or through segmentation in Freesurfer (whole brain: $r=0.0228, p=0.9140, N=25$; primary auditory cortex: $r=$ $-0.0430, p=0.8385, N=25$; anterior prefrontal cortex: $r=$ $-0.0231, p=0.9128, N=25)$.

\section{Discussion}

We report here several findings pertaining to the principles of human cortical organization and its variability across individuals. Specifically, we found a strong and consistent reciprocal relationship between the gray matter volume of primary sensory cortices and that of anterior prefrontal cortex. Our study showed that individuals with larger primary visual cortex had larger primary auditory cortex but smaller anterior prefrontal cortex, regardless of differences in their overall brain size. This inverse structural relationship formed along two separate pathways, in that the dorsal versus ventral halves of primary visual cortex shared covariance with the corresponding but not the opposite half of associated visual cortices (V2, V3) and exhibited focal negative correlations with the dorsolateral versus ventromedial parts of anterior prefrontal cortex. Surprisingly, this anatomical trade-off was specific to primary sensory cortices, as it was not observed for other retinotopic visual cortices (V2, V3) but was also observed for primary auditory cortex. Moreover, while one might expect a positive correlation between the whole-brain gray matter volume and the volume of its components, instead we found a striking anticorrelation for primary visual cortex: individuals with larger brains tended to have smaller primary visual cortices. In contrast, anterior prefrontal cortex was the single most enlarged region in a larger brain.

In contrast to a simple notion that different components of the human cortex scale with each other, our findings revealed an intriguing and hitherto unknown principle of human cortical organization-a trade-off between the sizes of primary sensory cortices and prefrontal cortex across individuals. Notably, while the behavioral consequences of variability in size of different cortical regions have received great research interest recently (Magu- ire et al., 2000; Draganski et al., 2004; Fleming et al., 2010; Schwarzkopf et al., 2011), relatively few studies have investigated whether and how the anatomical variability across different cortical regions are governed by a general principle. Previous work shows that the gray matter volumes of related structures early in the visual system (Andrews et al., 1997), or of corresponding regions in different hemispheres (Mechelli et al., 2005) covary in size across individuals, perhaps as a result of experience-related plasticity. Here, we instead showed that distinct brain regions could either scale with or scale against each other depending on their functional roles. Specifically, we found that the volumes of primary sensory (visual, auditory) cortices scaled with each other, whereas they scaled against the volume of anterior prefrontal cortex. As anterior prefrontal cortex is a key cortical region that supports high-order cognitive functions advanced in humans (Ramnani and Owen, 2004; Amodio and Frith, 2006), this anatomical trade-off hints toward a reciprocal link, behaviorally or functionally, between the two fundamental cognitive domains - basic sensation and high-order cognition.

As for other organs, the specification and development of the cerebral cortex is controlled by an interplay between extrinsic (epigenetic) and intrinsic (genetic) factors (Rakic, 1988; Neve and Bear, 1989; Miyashita-Lin et al., 1999). Intriguingly, anterior prefrontal cortex and primary visual cortex are located at the two opposite ends of the brain (anterior and posterior, respectively). In mice, the two genes Emx 2 and Pax6 are expressed in opposing gradients along the anterior-posterior axis. In Emx2 mutant mice, anterior areas are expanded, whereas posterior areas are contracted, while the opposite is the case for Pax6 mutant mice (Bishop et al., 2000). It is possible that human cortical arealization may also be regulated by genes with contrasting expression along the anterior-posterior axis and probably through separated pathways (e.g., anterior-ventromedial-posterior, anterior-dorsolateral-posterior). Consistent with this, the occipital pole (V1) and the frontal pole (aPFC) mature the earliest during human cortical development (Gogtay et al., 2004). Together, we speculate that the reciprocal structural relationship we observed between primary visual cortex and anterior prefrontal cortex might result from the individual variations in gene expression levels, possibly regulated by environmental interventions during development. Since the structural relationship was driven by surface area rather than cortical thickness, it would be of interest for further investigation to focus on how the dissociation in genetic influences on cortical surface area versus thickness (Panizzon et al., 2009) may contribute to the negative correlation between primary visual cortex and anterior prefrontal cortex.

The positive correlation that we observed between the gray matter volume of primary visual cortex and primary auditory cortex, however, may reflect a combined effect of experiencerelated plasticity and anatomical homogeneity. In real-world situations, our senses are usually stimulated simultaneously by a common source. Multisensory experience not only affects multisensory convergence areas but also unisensory cortices (Watkins et al., 2006; Noesselt et al., 2007; Driver and Noesselt, 2008). For example, visual signals can be decoded with multivariate pattern analysis from fMRI activity in primary auditory cortex (Meyer et al., 2010), and audiovisual temporal synchronization can affect activity in both primary visual and primary auditory cortex (Noesselt et al., 2007). Consistent with these functional interactions, our study suggests a structural covariance between primary sensory cortices, which may result from the frequent occurrence of audiovisual costimulation in everyday life or alternatively may reflect the coexpression or coregulation between genes. 
Intriguingly, the principles of cortical organization that we observed across a large group of humans are recapitulated by evolutionary milestones in brain and cognitive development. Over the last 2 million years, the hominid brain has increased in size more than threefold; but compared with allometric predictions from other species, humans have relatively smaller primary visual cortex (de Sousa et al., 2010). In contrast, as evolution progresses, anterior prefrontal cortex grows substantially in size (Semendeferi et al., 2001) despite the lack of change in overall frontal lobe size (Semendeferi et al., 2002). Strikingly parallel to these evolutionary trends across different species, our study showed that, within modern humans, brain size covaried negatively with the size of primary visual cortex but covaried positively with the size of anterior prefrontal cortex. Since genes regulating brain size undergo adaptive evolution even in modern humans (Evans et al., 2005; Mekel-Bobrov et al., 2005), the anatomical reciprocal relationship we observed may have ecological significance and reflect the gene expression divergences associated with this adaptive evolution.

Our study revealed possible new principles of human cortical organization. The pairing between the expansion of anterior prefrontal cortex and the contraction of primary sensory cortices reflects a common ground for the formation of anatomically and phylogenetically remote cortical regions, and suggests the existence of a reciprocal link between high-order cognition and low-level sensation.

\section{References}

Amodio DM, Frith CD (2006) Meeting of minds: the medial frontal cortex and social cognition. Nat Rev Neurosci 7:268-277.

Andrade A, Paradis AL, Rouquette S, Poline JB (1999) Ambiguous results in functional neuroimaging data analysis due to covariate correlation. Neuroimage 10:483-486.

Andrews TJ, Halpern SD, Purves D (1997) Correlated size variations in human visual cortex lateral geniculate nucleus and optic tract. J Neurosci 17:2859-2868.

Ashburner J (2007) A fast diffeomorphic image registration algorithm. Neuroimage 38:95-113.

Ashburner J, Friston KJ (2000) Voxel-based morphometry-the methods. Neuroimage 11:805-821.

Bishop KM, Goudreau G, O'Leary DD (2000) Regulation of area identity in the mammalian neocortex by emx2 and pax6. Science 288:344-349.

Dekaban AS (1978) Changes in brain weights during the span of human life: relation of brain weights to body heights and body weights. Ann Neurol 4:345-356.

Desikan RS, Ségonne F, Fischl B, Quinn BT, Dickerson BC, Blacker D, Buckner RL, Dale AM, Maguire RP, Hyman BT, Albert MS, Killiany RJ (2006) An automated labeling system for subdividing the human cerebral cortex on MRI scans into gyral based regions of interest. Neuroimage 31:968-980.

de Sousa AA, Sherwood CC, Mohlberg H, Amunts K, Schleicher A, MacLeod CE, Hof PR, Frahm H, Zilles K (2010) Hominoid visual brain structure volumes and the position of the lunate sulcus. J Hum Evol 58:281-292.

Dougherty RF, Koch VM, Brewer AA, Fischer B, Modersitzki J, Wandell BA (2003) Visual field representations and locations of area V1/2/3 in human visual cortex. J Vis 3:586-598.

Draganski B, Gaser C, Busch V, Schuierer G, Bogdahn U, May A (2004) Changes in gray matter induced by training. Nature 427:311-312.

Driver J, Noesselt T (2008) Multisensory interplay reveals crossmodal influences on "sensory-specific" brain regions neural responses and judgments. Neuron 57:11-23.

Evans PD, Gilbert SL, Mekel-Bobrov N, Vallender EJ, Anderson JR, VaezAzizi LM, Tishkoff SA, Hudson RR, Lahn BT (2005) Microcephalin a gene regulating brain size continues to evolve adaptively in humans. Science 309:1717-1720.

Fischl B, Sereno MI, Dale AM (1999) Cortical surface-based analysis II: inflation flattening and a surface-based coordinate system. Neuroimage 9:195-207.

Fleming SM, Weil RS, Nagy Z, Dolan RJ, Rees G (2010) Relating introspective accuracy to individual differences in brain structure. Science 329:1541-1543.

Gogtay N, Giedd JN, Lusk L, Hayashi KM, Greenstein D, Vaituzis AC, Nugent
TF 3rd, Herman DH, Clasen LS, Toga AW, Rapoport JL, Thompson PM (2004) Dynamic mapping of human cortical development during childhood through early adulthood. Proc Natl Acad Sci U S A 101:8174-8179.

Hagmann P, Cammoun L, Gigandet X, Meuli R, Honey CJ, Wedeen VJ, Sporns O (2008) Mapping the structural core of human cerebral cortex. PLoS Biol 6:e159.

Hayasaka S, Phan KL, Liberzon I, Worsley KJ, Nichols TE (2004) Nonstationary cluster-size inference with random field and permutation methods. Neuroimage 22:676-687.

Maguire EA, Gadian DG, Johnsrude IS, Good CD, Ashburner J, Frackowiak RS, Frith CD (2000) Navigation-related structural change in the hippocampi of taxi drivers. Proc Natl Acad Sci U S A 97:4398-4403.

Maldjian JA, Laurienti PJ, Kraft RA, Burdette JH (2003) An automated method for neuroanatomic and cytoarchitectonic atlas-based interrogation of fMRI data sets. Neuroimage 19:1233-1239.

Mechelli A, Friston KJ, Frackowiak RS, Price CJ (2005) Structural covariance in the human cortex. J Neurosci 25:8303-8310.

Mekel-Bobrov N, Gilbert SL, Evans PD, Vallender EJ, Anderson JR, Hudson RR, Tishkoff SA, Lahn BT (2005) Ongoing adaptive evolution of ASPM a brain size determinant in Homo sapiens. Science 309:1720-1722.

Meyer K, Kaplan JT, Essex R, Webber C, Damasio H, Damasio A (2010) Predicting visual stimuli on the basis of activity in auditory cortices. Nat Neurosci 13:667-668.

Miyashita-Lin EM, Hevner R, Wassarman KM, Martinez S, Rubenstein JL (1999) Early neocortical regionalization in the absence of thalamic innervation. Science 285:906-909.

Morosan P, Rademacher J, Schleicher A, Amunts K, Schormann T, Zilles K (2001) Human primary auditory cortex: cytoarchitectonic subdivisions and mapping into a spatial reference system. Neuroimage 13:684-701.

Neve RL, Bear MF (1989) Visual experience regulates gene expression in the developing striate cortex. Proc Natl Acad Sci U S A 86:4781-4784.

Noesselt T, Rieger JW, Schoenfeld MA, Kanowski M, Hinrichs H, Heinze HJ, Driver J (2007) Audiovisual temporal correspondence modulates human multisensory superior temporal sulcus plus primary sensory cortices. J Neurosci 27:11431-11441.

Pallas SL (2001) Intrinsic and extrinsic factors that shape neocortical specification. Trends Neurosci 24:417-423.

Panizzon MS, Fennema-Notestine C, Eyler LT, Jernigan TL, Prom-Wormley E, Neale M, Jacobson K, Lyons MJ, Grant MD, Franz CE, Xian H, Tsuang M, Fischl B, Seidman L, Dale A, Kremen WS (2009) Distinct genetic influences on cortical surface area and cortical thickness. Cereb Cortex 19:2728-2735.

Rakic P (1988) Specification of cerebral cortical areas. Science 241:170-176.

Ramnani N, Owen AM (2004) Anterior prefrontal cortex: insights into function from anatomy and neuroimaging. Nat Rev Neurosci 5:184-194.

Roth G, Dicke U (2005) Evolution of the brain and intelligence. Trends Cogn Sci 9:250-257.

Rubin N, Nakayama K, Shapley R (1996) Enhanced perception of illusory contours in the lower versus upper visual hemifields. Science 271:651-653.

Schoenemann PT (2006) Evolution of the size and functional areas of the human brain. Annu Rev Anthropol 35:379-406.

Schwarzkopf DS, Song C, Rees G (2011) The surface area of human V1 predicts the subjective experience of object size. Nat Neurosci 14:28-30.

Semendeferi K, Armstrong E, Schleicher A, Zilles K, Van Hoesen GW (2001) Prefrontal cortex in humans and apes: a comparative study of area 10 . Am J Phys Anthropol 114:224-241.

Semendeferi K, Lu A, Schenker N, Damasio H (2002) Humans and great apes share a large frontal cortex. Nat Neurosci 5:272-276.

Sereno MI, Dale AM, Reppas JB, Kwong KK, Belliveau JW, Brady TJ, Rosen BR, Tootell RB (1995) Borders of multiple visual areas in human revealed by functional magnetic resonance imaging. Science 268:889-893.

Stensaas SS, Eddington DK, Dobelle WH (1974) The topography and variability of the primary visual cortex in man. J Neurosurg 40:747-755.

Velicer WF, Jackson DN (1990) Component analysis versus common factor analysis: some issues in selecting an appropriate procedure. Multivar Behav Res 25:1-28.

Wandell BA, Dumoulin SO, Brewer AA (2007) Visual field maps in human cortex. Neuron 56:366-383.

Watkins S, Shams L, Tanaka S, Haynes JD, Rees G (2006) Sound alters activity in human V1 in association with illusory visual perception. Neuroimage 31:1247-1256. 